\title{
FREE CONVECTION OF HEAT TRANSFER IN FLOW PAST A SEMI-INFINITE FLAT PLATE IN TRANSVERSE MAGNETIC FIELD WITH HEAT FLUX
}

\author{
${ }^{1}$ Geetha, P., ${ }^{2}$ M.B.K. Moorthy and ${ }^{3}$ M. Dakshinamoorthy \\ ${ }^{1}$ Department of Mathematics, Bannari Amman Institute of Technology, Sathyamangalam-638 401, India \\ ${ }^{2}$ Department of Mathematics, Institute of Road and Transport, Erode-638 316, India \\ ${ }^{3}$ Department of Physics, Institute of Road and Transport, Erode-638 316, India
}

Received 2014-05-17; Revised 2014-06-25; Accepted 2014-07-04

\begin{abstract}
A steady two-dimensional MHD free convection of heat transfer in flow past a semi-infinite flat plate in transverse magnetic field with heat flux has been examined. The governing partial differential equation are non-dimensionalised and transformed into a system of nonlinear ordinary differential similarity equations, in a single independent variable $\eta$ and using Runge-Kutta Gill method with shooting technique. The velocity and temperature distributions are discussed numerically and presented through graphs. Skin-friction coefficient and the Nusselt number are derived, discussed numerically and their numerical values for various values of physical parameter are presented through tabular form. The effects of magnetic parameter, permeability parameter and Prandtl number on the velocity and temperature profiles were displayed graphically for different values of parameters entering into the problem. Significant changes were obtained in heat transfer coefficient due to the parameters. In addition, the skin friction coefficient and Nusselt number were shown in tabular form.
\end{abstract}

Keywords: Steady, Boundary Layer, MHD, Free Convection, Heat Flux

\section{INTRODUCTION}

Owing to their numerous applications in industrial manufacturing process, the problem of heat transfer in fluid-saturated porous media finds applications in a variety of engineering processes such as heat exchanger devices, petroleum reservoirs, chemical catalytic reactors and processes, geothermal and geophysical engineering, moisture migration in a fibrous insulation and nuclear waste disposal and others. Some of the applications areas are hot rolling, paper production, metal spinning, drawing plastic films, glass blowing, continuous casting of metals and spinning of fibers. In particular the extradite from the die is drawn and simultaneously stretched into a sheet, which is then solidified through quenching or gradual cooling by direct contact with example. Lai and Kulacki (1990) used the series expansion water. Annealing and thinning of copper wires is another

method to investigate coupled heat and mass transfer in natural connection from a sphere in a porous medium.

Magnetohydrodynamics flows have applications in meteorology, solar physics, cosmic fluid dynamics, astrophysics, geophysics and in the motion of earth's core. In addition from the technological point of view, MHD free convection flows have significant applications in the field of stellar and planetary magneto spheres, aeronautical plasma flows, chemical engineering and electronics. An excellent summary of applications is given by (Huges and Young, 1966; Raptis, 1986) studied mathematically the case of time varying two dimensional natural convective flow of an incompressible, electrically conducting fluid along an infinite vertical plate embedded in a porous medium.

Helmy (1998) analyzed MHD unsteady free convection flow past a vertical porous plate embedded Corresponding Author: Geetha, P., Department of Mathematics, Bannari Amman Institute of Technology, Sathyamangalam-638 401 
in a porous medium. Elabashbeshy (1997) studied heat and mass transfer along a vertical plate in the presence of magnetic field.

In all the studies mentioned above, the heat due to viscous dissipation is neglected. Gebhart (1962) has shown the importance of viscous dissipation heat in free convection flow in the case of isothermal and constant heat flux at the plate. Gebhart and Mollendorf (1969) considered the effects of viscous dissipation for external natural convection flow over a surface. Soundalgekar (1972) analyzed viscous dissipative heat on the two-dimensional unsteady free convective flow past an infinite vertical porous plate when the temperature oscillates in time and there is constant suction at the plate. Israel Cookey et al. (2003) investigated the influence of viscous dissipation and radiation on unsteady MHD free convection flow past an infinite heated vertical plate in a porous medium with time dependent suction. The governing equations are solved numerically using the Runge-kutta method with shooting technique. The effects of various governing parameters on the velocity, temperature, skin-friction coefficient and Nusselt number are shown in Fig. and Table 1 and analyzed in detail.

\section{MATHEMATICAL ANALYSIS}

A steady, two dimensional, incompressible flow of a viscous fluid on a continuous flat surface, issuing from a slot and moving with a constant velocity U0 in a fluid at rest, in the presence of a transverse magnetic field of strength B0 is considered.

Let the $x$-axis be taken along the sheet in the direction of motion of the sheet and y-axis normal to it with velocity components $\mathrm{u}$ and $\mathrm{v}$ directed along their axes respectively. A uniform magnetic field is applied in the direction perpendicular to the plate. The fluid is assumed to be slightly conducting and hence the magnetic Reynolds number is much less than unity and the induced magnetic field is negligible in comparison with the applied magnetic field. If $\sigma$ is the electrical conductivity of the fluid then the flow and heat transfer are given by the following equations.

Continuity Equation 1:

$$
\frac{\partial u}{\partial x}+\frac{\partial v}{\partial y}=0
$$

Momentum Equation 2: $u \frac{\partial u}{\partial x}+v \frac{\partial u}{\partial y}=v \frac{\partial^{2} u}{\partial y^{2}}-\sigma \frac{B_{0}^{2} u}{\rho}-\frac{v}{k^{\prime}} u$

Energy Equation 3:

$u \frac{\partial T}{\partial x}+v \frac{\partial T}{\partial y}=\alpha \frac{\partial^{2} T}{\partial y^{2}}$

The boundary conditions are Equation 4:

$$
\begin{array}{ll}
u=U_{0}, & v=0, \quad \frac{\partial T}{\partial y}=-\frac{q_{w}}{k} \quad \text { at } y=0 \\
u \rightarrow 0, & T \rightarrow T_{\infty}, \quad \text { as } y \rightarrow \infty
\end{array}
$$

Here $U O$ is the uniform velocity of the plate and qw is the heat flux per unit area. $u, v$ are the velocity components in $\mathrm{x}, \mathrm{y}$ directions respectively, $\rho$ the density of the fluid, k' the permeability of the porous medium, $\mathrm{T}$ the temperature of the fluid, $v$ the kinematic viscosity, $\alpha=k / \rho c p$ the thermal diffusivity, $\mathrm{cp}$ the specific heat at constant pressure, $\mathrm{k}$ the thermal conductivity of the fluid, B0 the magnetic induction, $\sigma$ the electrical conductivity of the fluid.

The Equation (2 and 3) are coupled, parabolic and nonlinear partial differential equations and hence analytical solution is not possible. Therefore numerical technique is employed to obtain the required solution. Numerical computation is greatly facilitated by non-dimensionalization of the equations. Proceeding with the analysis, we introduce the following similarity transformations and dimensionless variables which will convert the partial differential equation from two independent $(\mathrm{x}, \mathrm{y})$ variables to a system of coupled, non-linear ordinary differential equations in a single variable $\eta$. i.e., coordinate normal to the plate.

In order to write the governing equations and the boundary conditions in dimensionless for the nondimensional quantities are introduced by the stream function $\psi$, defined by Equation 5:

$u=\frac{\partial \psi}{\partial y}$ and $v=-\frac{\partial \psi}{\partial x}$

Which satisfy the equation of continuity and making the following substitution (assuming $\mathrm{f}, \theta$ to be the functions of $\eta$ only): 


$$
\begin{aligned}
& \psi=\sqrt{v x U_{0}} f \\
& \eta=y \sqrt{\frac{U_{0}}{v x}} \\
& v=\frac{1}{2} \sqrt{\frac{v U_{0}}{x}}\left(\eta f^{\prime}-f\right) \\
& u=U_{0} f \text {. } \\
& \theta(\eta)=\frac{k\left(T-T_{\infty}\right)}{q_{w}} \sqrt{\frac{U_{0}}{v x}} \\
& \operatorname{Pr}=\frac{\mu c_{p}}{k}(\operatorname{Pr} \text { andtl number }) \\
& \left.M=\frac{m^{2}}{\operatorname{Re}} \text { (Magnetic field parameter }\right) \\
& m^{2}=\frac{\sigma B_{0}^{2} x^{2}}{\mu}(m \text { is the Hartman number }) \\
& \operatorname{Re}=\frac{U_{0} x}{v}(\operatorname{Re} y \text { nolds number }) \\
& K=\frac{v x}{k^{\prime} U_{0}}(\text { Permeability parameter })
\end{aligned}
$$

One finds that the boundary layer Equations (1 to 3) become:

$$
\begin{aligned}
& f^{\prime \prime \prime}+\frac{1}{2} f f^{\prime \prime}-M f^{\prime}-K f^{\prime}=0 \\
& \theta^{\prime \prime}+\frac{\operatorname{Pr}}{2}\left(f \theta^{\prime}-f^{\prime} \theta\right)=0
\end{aligned}
$$

With boundary conditions Equation 9:

$$
\begin{array}{rrr}
f(0)=0, & f^{\prime}(0)=1, \quad \theta^{\prime}(0)=-1 \quad \text { at } \eta=0 \\
f^{\prime}(\infty)=0, \theta(\infty)=0 & \text { as } \eta \rightarrow \infty
\end{array}
$$

Equations (7), (8) subject to the boundary conditions (9) were solved numerically on a computer for different values of magnetic parameter for air using shooting method. The effects of variation of $\mathrm{M}$ and $\mathrm{K}$ on temperature profiles have been plotted. Numerical values off $(\eta)$ and $f^{\prime}(\eta)$ for different $\mathrm{M}$ values have been calculated and they are presented in a tabular form.

The skin-friction coefficient and Nusselt number are important physical parameters for this type of boundary layer flow.

Knowing the velocity field, the skin-friction coefficient at the plate can be obtained, which in nondimensional form is given by:

$$
C_{f}=2(\operatorname{Re})^{\frac{-1}{2}} f^{\prime \prime}(0)
$$

Knowing the temperature field, the rate of heat transfer coefficient can be obtained, which in non-dimensional form, in terms of the Nusselt number, is given by:

$$
N u=(\operatorname{Re})^{\frac{-1}{2}} \theta^{\prime}(0)
$$

\section{SOLUTION OF THE PROBLEM}

The set of coupled non-linear governing boundary layer Equation (7 and 8) together with the boundary conditions (9) are solved numerically by using RungeKutta Gill method along with the shooting technique. First of all, higher order non-linear differential equations (7) to (8) are converted into simultaneous linear differential equations of first order and they are further transformed into initial value problem by applying the shooting technique. The resultant initial value problem is solved by employing Runge-Kutta Gill method. The step size $\Delta \eta=0.05$ is used to obtain the numerical solution with five decimal place accuracy as the criterion of convergence. From the process of numerical computation, the skin-friction coefficient and the Nusselt number, which are respectively proportional to $f^{\prime}(0)$ and- $\theta^{\prime}(0)$ are also sorted out and their numerical values are presented in a tabular form. Extensive calculations have been performed to obtain the flow and temperature fields for a wide range of parameters $0.5 \leq \mathrm{M} \leq 2,0 \leq \mathrm{K} \leq 2$ and $1 \leq \mathrm{Pr} \leq 5$.

\section{RESULTS}

Table 1 shows numerical values of magnetic field parameter effects on $\left(f^{\prime} 0\right)$ and $\theta^{\prime}(0)$. The profiles for velocity and temperature are shown in Fig. 1-5 respectively with various values of the parameters.

\section{DISCUSSION}

We now discuss the results. As a result of the numerical calculations, the dimensionless velocity and temperature distributions for the flow under consideration are obtained and their behavior have been discussed for variations in the governing parameters viz., magnetic field parameter $\mathrm{M}$, Permeability parameter K, Prandtl number Pr. The influence of magnetic field parameter $\mathrm{M}$ on the velocity is presented in Fig. 1. It is observed that there is a decrease in the velocity as the magnetic field parameter $M$ increases. This result qualitatively agrees with the expectations, since the magnetic field exerts a retarding force on the free convection flow. Also, as $\mathrm{M}$ increases, the peak values of the velocity decreases rapidly near the porous plate and their decays smoothly to the stream velocity. 
Figure 2 presents typical velocity profiles in the boundary layer for various values of the permeability parameter $\mathrm{K}$ while all other parameters are kept at some fixed values. The parameter $\mathrm{K}$ is inversely proportional to the actual permeability $K^{\prime}$ of the porous medium. An increase in $\mathrm{K}$ will therefore increase the resistance of the porous medium which will tend to decelerate the flow and reduce the velocity. The fluid velocity decreases as the permeability parameter $\mathrm{K}$ increases. Also as $\mathrm{K}$ increases, the peak values of the velocity decreases rapidly near the plate and decreases smoothly to approach the free stream velocity.

Figure (3a and b) illustrate the velocity and temperature profiles for different values of the Prandtl number Pr. The Prandtl number defines the ratio of momentum diffusivity to the thermal diffusivity. The numerical results show that the effect of increasing values of Prandtl number results in a decreasing velocity. From Fig. 3b, it is observed that an increase in the Prandtl number results a decrease of the thermal boundary layer thickness and in general lower average temperature within the boundary layer. The reason is that smaller values of $\operatorname{Pr}$ are equivalent to increasing the thermal conductivities and therefore heat is able to diffuse away from the heated plate more rapidly than for higher values of Pr. Hence in the case of smaller Prandtl numbers as the boundary layer is thicker and the rate of heat transfer is reduced.

Figure 4 presents the temperature profile for various values of magnetic field parameter $M$ while all other parameters are kept at some fixed values. An increase in Magnetic field parameter $M$ results in increase in temperature profile.

It is observed that from Fig. 5 the increase in the permeability parameter $\mathrm{K}$ increases the temperature profile.

The effects of various governing parameters on the skin friction coefficient $\mathrm{Cf}$ and Nusselt number $\mathrm{Nu}$ are shown in the Table 1. It is observed from the Table $\mathbf{1}$ that as $\mathrm{M}$ and $\mathrm{K}$ increases, there is a fall in the skin-friction coefficient but there is an increase in Nusselt number. As the Prandtl number increases there is no change in the skin-friction coefficient but there is a decrease in the Nusselt number.

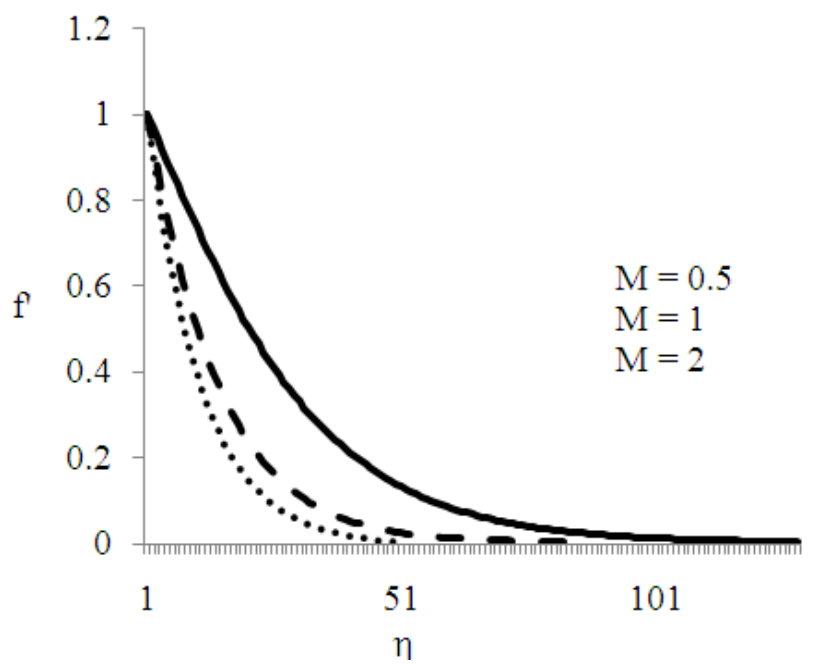

Fig. 1. Effect of Magnetic field parameter $M$ on non-dimensional velocity f'

Table 1: Magnetic field parameter effect on $\mathrm{f}^{\prime}(0)$ and $\theta(0)$

\begin{tabular}{lllll}
\hline $\mathrm{M}$ & $\mathrm{K}$ & $\operatorname{Pr}$ & $\mathrm{f}^{\prime \prime}(0)$ & $\theta^{\prime}(0)$ \\
\hline 0.5 & 0.5 & 0.71 & -0.4438 & 1.6466 \\
1.0 & 0.5 & 0.71 & -1.0828 & 2.1758 \\
2.0 & 0.5 & 0.71 & -1.4733 & 2.8471 \\
0.5 & 0.5 & 0.71 & -0.4439 & 1.6458 \\
0.5 & 1.0 & 0.71 & -1.0828 & 2.1729 \\
0.5 & 2.0 & 0.71 & -1.4734 & 2.8314 \\
0.5 & 0.5 & 1.00 & -0.4444 & 1.3312 \\
0.5 & 0.5 & -0.4444 & 1.1647 \\
0.5 & 0.5 & 5.00 & -0.4444 & 0.5370 \\
0.5 & 0.5 & 100 & -0.4444 & 0.3721 \\
\hline
\end{tabular}


Geetha P. et al. / American Journal of Applied Sciences 11 (9): 1480-1485, 2014

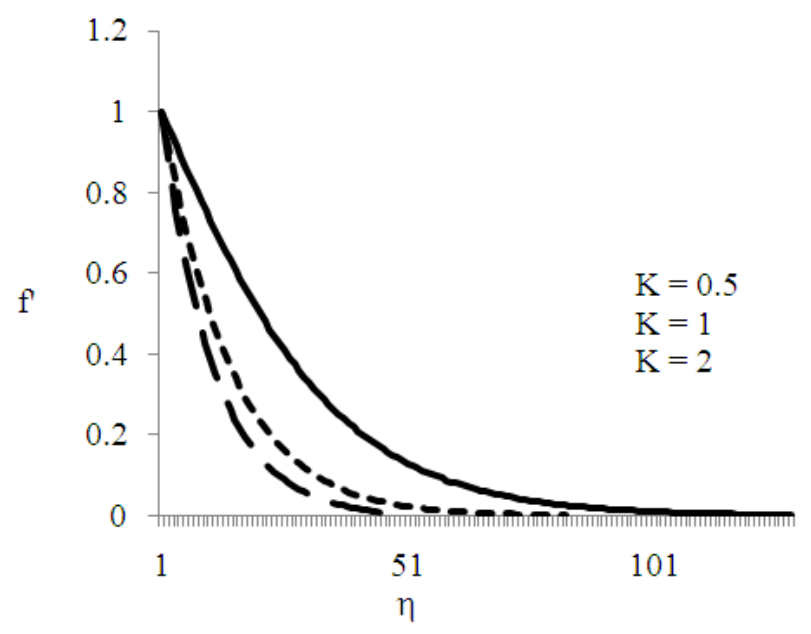

Fig. 2. Effect of Permeability parameter $\mathrm{K}$ on non-dimensional velocity $\mathrm{f}^{\prime}$
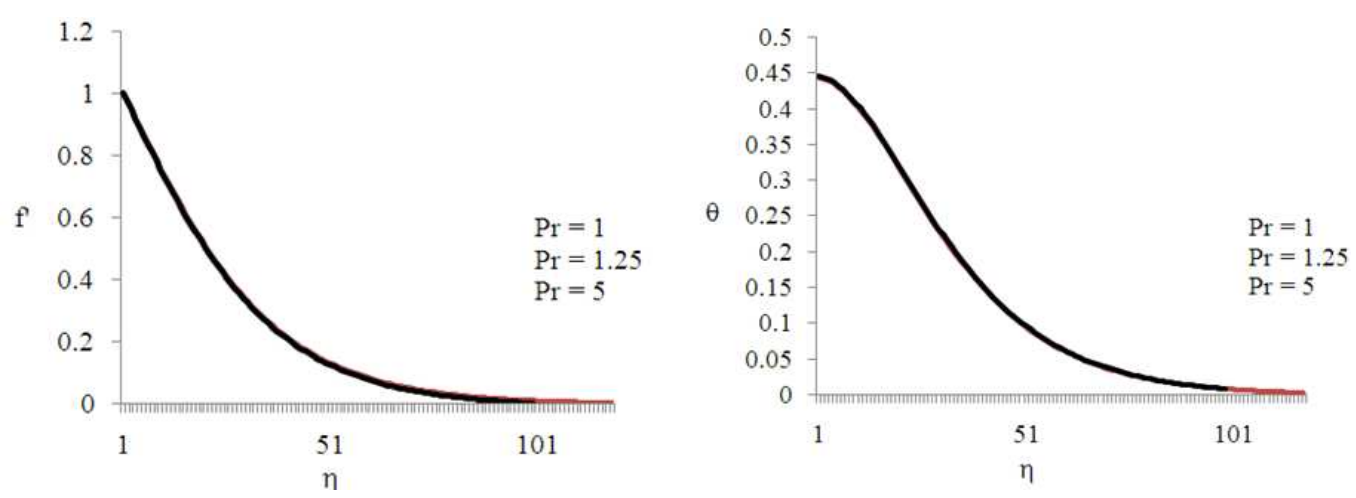

Fig. 3(a). Effect of Prandtl number Pr on non-dimensional velocity f' (b) Effect of Prandtl number Pr on non-dimensional velocity temperature $\theta$

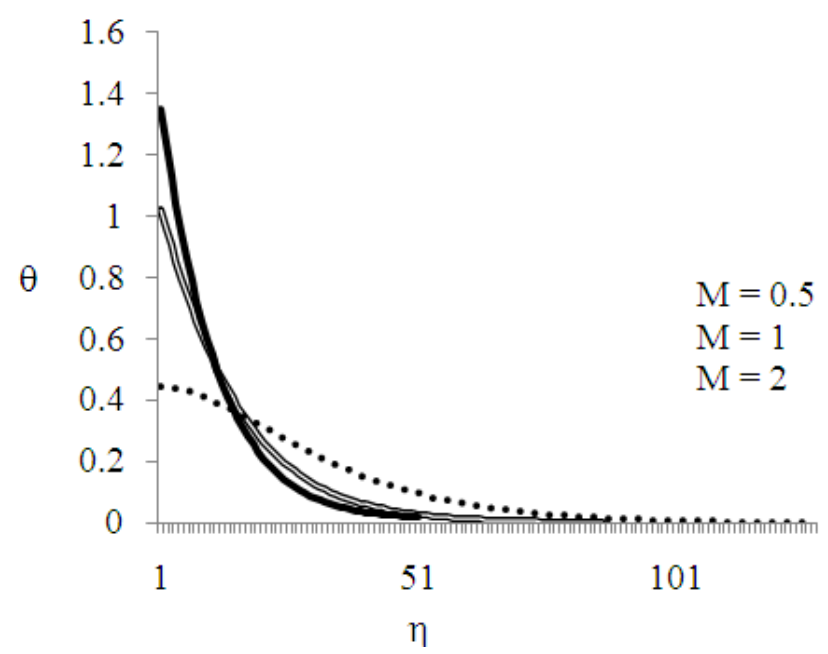

Fig. 4. Effect of Magnetic field parameter $M$ on non-dimensional temperature $\theta$ 


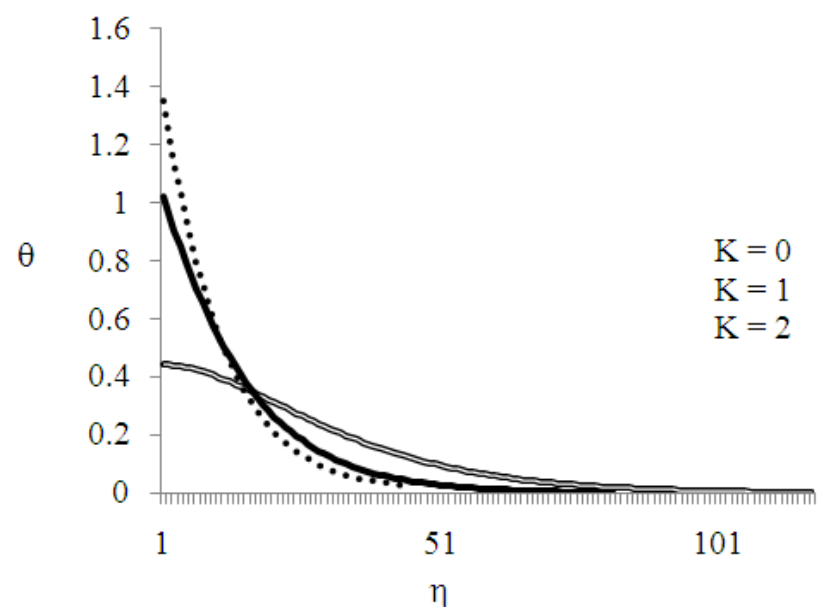

Fig. 5. Effect of Permeability parameter $\mathrm{K}$ on non-dimensional temperature $\theta$

\section{CONCLUSION}

Using the similarity transformation a set of ordinary differential equations has been derived for the conservation of mass and momentum in the boundary layer. These nonlinear, coupled differential equations have been solved physically valid boundary conditions using Runge-kutta method along with shooting technique. The conclusions of the study are as follows:

- The velocity decreases with an increase in the magnetic parameter and permeability parameter

- A positive increase in Prandtl number is shown to reduce the velocity and temperature in the flow

- The temperature decreases with an increase in magnetic parameter and permeability parameter

- An increase in $\mathrm{M}$ and $\mathrm{K}$ leads to fall in the skin-friction coefficient but there is an increase in Nusselt number

\section{ACKNOWLEDGMENT}

The authors render their heartfelt thanks to the Chairman, Trustee, Chief Executive, Principal for their support to do this study. The authors wish to thank the Director IRT, Chennai and the Principal IRTT, for their generous help.

\section{REFERENCES}

Elabashbeshy, E.M.A., 1997. Heat and mass transfer along a vertical plate with variable temperature and concentration in the presence of magnetic field. Int. J. Eng. Sci., 34: 515-522.
Gebhart, B. and J. Mollendorf, 1969. Viscous dissipation in external natural convection flows. J. Fluid. Mech., 38: 97-107. DOI: 10.1017/S0022112069000061

Gebhart, B., 1962. Effects of viscous dissipation in natural convection. J. Fluid Mech., 14: 225-232. DOI: $10.1017 /$ S0022112062001196

Helmy, K.A., 1998. MHD unsteady free convection flow past a vertical porous plate. ZAMM-J. Applied Math. Mech. /Zeitschrift für Angewandte Mathemat. Mech., 78: 255-270. DOI: 10.1002/(SICI)1521-4001

Huges, W.F. and F.J. Young, 1966. The Electro-Magneto Dynamics of Fluids. John Wiley and Sons, New York.

Israel Cookey, C., A. Ogulu and V.B. Omubo-Pepple, 2003. Influence of viscous dissipation and radiation on unsteady MHD free-convection flow past an infinite heated vertical plate in a porous medium with time-dependent suction. Int. J. Heat Mass Transfer, 46: 2305-2311. DOI: 10.1016/S00179310(02)00544-6

Lai, F.C. and F.A. Kulacki, 1990. Coupled heat and mass transfer from a sphere buried in an infinite porous medium. Int. J. Heat Mass Trans., 33: 209-215. DOI: 10.1016/0017-9310(90)90155-N

Raptis, A., 1986. Flow through a porous medium in the presence of a magnetic field. Int. J. Energy Res., 10: 97-101. DOI: 10.1002/er.4440100112

Soundalgekar, V.M., 1972. Viscous dissipation effects on unsteady free convective flow past an infinite, vertical porous plate with constant suction. Int. J. Heat Mass Transfer, 15: 1253-1261. DOI: 10.1016/0017-9310(72)90189-5 